\title{
An Aphid Facultative Symbiont Suppresses Plant Defense by Manipulating Aphid Gene Expression in Salivary Glands
}

\author{
Qinyang Wang ${ }^{1}$, Erliang Yuan ${ }^{1}$, Xiaoyu Ling ${ }^{1}$, keyan zhu-salzman ${ }^{2}$, Huijuan Guo ${ }^{1}$, Feng \\ $\mathrm{Ge}^{1}$, and Sun Yucheng ${ }^{1}$ \\ ${ }^{1}$ Institute of Zoology Chinese Academy of Sciences \\ ${ }^{2}$ Texas A\&M University
}

April 28, 2020

\begin{abstract}
Aphids often carry facultative symbionts to achieve diverse advantages. Serratia symbiotica, one of facultative endosymbionts, increases aphid tolerance to heat. However, whether it benefits aphid colonization on host plants is yet to be determined. In the current study, we found that Acyrthosiphon pisum harboring S. symbiotica had longer feeding duration on Medicago truncatula than Serratia-free aphids. Contrastingly, Serratia-free aphids triggered higher accumulation of ROS, jasmonic acid and salicylic acid responsive genes and cytosolic $\mathrm{Ca} 2+$ elevations than Serratia-infected aphids. Transcriptomic analysis of salivary glands indicated that a histidine-rich Ca2+-binding protein-like gene (ApHRC) was expressed more highly in the salivary gland of Serratia-infected aphids than that of Serratia-free aphids. Once ApHRC was silenced, Serratia-infected aphids also displayed shorter phloem-feeding duration and caused $\mathrm{Ca} 2+$ elevation and ROS accumulation in plants. Our results suggest that ApHRC, a potential effector up-regulated by S. symbiotica in the salivary glands, evaded plant defense response by suppressing Ca2 + elevation and ROS accumulation, allowing colonization of aphids. This study has provided a revolutionary insight into how facultative symbionts facilitate aphid colonization and adaption to host plants.
\end{abstract}

\section{Keywords}

Acyrthosiphon pisum ; Serratia symbiotica ; salivary gland; histidine-rich calcium-binding protein-like; calcium

\section{Introduction}

Insects are commonly associated with symbiotic microbes which could bring some advantages to themselves. The pea aphid Acyrthosiphon pisum, a model insect for endosymbiont studies, usually hosts one obligate symbiont and several facultative symbionts (Douglas \& Prosser, 1992; La Pena, Vandomme, \& Frago, 2014). Since phloem sap is a suboptimal diet for aphids due to imbalanced amino acids: carbohydrates proportions, the obligate symbiont, Buchnera aphidicola that resides in aphid bacteriocytes offers essential amino acids to aphids (Douglas, 1998). By contrast, facultative symbionts are usually not required for survival and reproduction of aphids but are able to improve their fitness particularly when they are confronted by various biotic and abiotic challenges. Studies indicate that some facultative symbionts increase heat tolerance (Doremus \& Olive, 2017), defense against the natural enemies and fungi (Lukasik, Van Asch, Guo, Ferrari, \& Godfray, 2013; Oliver, Russell, Moran, \& Hunter, 2003), and host plant adaptation of aphids (Leonardo \& Muiru, 2003). Previous research shows that an elicitor protein GroEL from B. aphidicolatriggers plant resistance against aphids (Chaudhary, Atamian, Shen, Briggs, \& Kaloshian, 2014). Hence, endosymbionts may modify the components of salivary proteins that modulate aphid feeding on plants. Very little is known, however, how facultative endosymbionts affect aphid feeding by altering aphid salivary proteins. 
Aphids use their stylets to obtain nutrients from phloem sieve elements of the host plants (Tjallingii \& Esch, 1993). While probing, aphids secrete saliva to inhibit plant defense response caused by wounding due to aphid feeding as well as clogging of the fluid in the phloem tissue usually induced by $\mathrm{Ca}^{2+}$ influx (Aidemark, Andersson, Rasmusson, \& Widell, 2009). Plants typically initiate rapid cytosolic $\mathrm{Ca}^{2+}$ sparks upon aphid probing (Vincent et al., 2017), resulting in the activation of local defenses including reactive oxygen species (ROS) and phytohormone signaling (Tian et al., 2019). ROS-induced cell death prevents the aphid stylet from reaching the phloem (Laitinen et al., 2017) and triggers the salicylic acid (SA) signaling pathway to confer the local plant resistance to aphids (Hogenhout \& Bos, 2011; Jaouannet et al., 2014). In addition, $\mathrm{Ca}^{2+}$ is also a long-distance signal that can be transmitted from injured cells to uninfected tissues, activating the wound-related systemic jasmonic acid (JA) signaling pathway (Farmer, Gasperini, \& Acosta, 2014). Both synergistic and antagonistic JA- and SA-regulated defense have been reported in plant responses to aphids (Moran and Thompson,2001; Moran et al., 2002; De Vos et al., 2005; Mewis et al., 2006; Pegadaraju, 2005). Conversely, $\mathrm{Ca}^{2+}$-binding proteins secreted by aphids into plant tissues have been shown to facilitate aphids feeding. For example, Armet, a salivary protein from the pea aphid sequesters $\mathrm{Ca}^{2+}$ to counteract the $\mathrm{Ca}^{2+}$-triggered occlusion in Vicia fabae, which prolongs aphid phloem feeding time (Wang et al., 2015). Effectors containing $\mathrm{Ca}^{2+}$-binding domains presumably could efficiently quench the cytosolic $\mathrm{Ca}^{2+}$ elevation of host plants, which consequently improves aphid feeding.

The facultative symbiont Serratia symbiotica increases heat tolerance of $A$. pisum, and enhances resistance to parasitoids and predators (Costopoulos, Kovacs, Kamins, \& Gerardo, 2014; Hopper et al., 2018; Montllor, Maxmen, \& Purcell, 2002), but little in known of whether and how S. symbiotica benefits aphid feeding. Previous studies showed that pea aphid clones collected from 11 legume plants differed in their facultative symbionts and infectious rate (Frantz, Calcagno, Mieuzet, Plantegenest, \& Simon, 2009; Henry et al., 2013; Simon et al., 2003), suggesting that host plant species impact the facultative symbionts associated with aphids. During July-August of 2015-2019, we intended to determine the prevalence of endosymbionts of pea aphids in Medicago sativa fields in Yinchuan city, Ningxia province, China, and identified 5 endosymbionts within 154 sampled pea aphids (Fig. S1). S. symbiotica had a relatively high infectious rate (85\%) in all sampled aphids, we hypothesized that infection of S. symbiotica may benefit the aphid growth on Medicagoplants by facilitating aphid feeding. To test the hypothesis, we monitored aphid feeding behavior, performed aphid salivary gland transcriptomic analysis in the presence and absence of S. symbiotica . Histidine-rich calciumbinding protein-like (GeneID: 103308203) (A. pisum HRC , ApHRC) that encodes a salivary protein was up-regulated by $S$. symbiotica. Further investigation indicated that ApHRC suppressed $\mathrm{Ca}^{2+}$ elevation, which otherwise would have induced the plant defense response. Our study has demonstrated that $S$. symbiotica infection can modify aphid salivary composition by modulating salivary gland gene expression, which suppressed plant defense and benefited aphid feeding.

\section{Methods}

Aphids and Plants. Pea aphids (A. pisum ) in red color used in experiments were collected from Medicago sativa fields in Yinchuan $\left(106.27^{\circ} \mathrm{N}, 38.47^{\circ} \mathrm{E}\right)$, Ningxia, China in 2015.Serratia -infected aphids were parthenogenetic descendants from a single isolated female. We established Serratia -free aphids by injecting ampicillin into Serratia -infected aphids. To minimize the effect of the antibiotic on experiments, we reestablishedSerratia -carrying aphids, namely Serratia -rebuilt aphids, by injecting hemolymph of Serratia -infected aphids intoSerratia -free (see Supplementary Information for details).M. truncatula plants (cv. A17) was kindly provided by Professor Wenhao Zhang, Institute of Botany, Chinese Academy of Sciences, China. Transgenic Nicotiana benthamiana overexpressing GCaMP3used to detect $\mathrm{Ca}^{2+}$ sparks, was kindly provided by Department of Cell \& Systems Biology, University of Toronto, Toronto, Canada (Defalco et al., 2017). Details of plant growth conditions were described in Supplementary Information .

Aphid Mean Relative Growth Rate (MRGR), Offspring Number and Development Duration. To measure pea aphid MRGR, $102^{\text {nd }}$ instar nymphs fromSerratia -free or Serratia -infected aphids were weighed with an automatic electrobalance before and after feeding on $M$. truncatula plants for 5 days. The MRGR was calculated as previously described (Leather \& Dixon, 1984): MRGR $=(\ln$ W2 - ln W1)/t, where 
$\mathrm{W} 1$ was the initial weight, $\mathrm{W} 2$ was the final weight, and $\mathrm{t}$ represented days between weighing, i.e. $\mathrm{t}=5$. Data were collected from 50 aphids.

For reproduction and developmental duration, one adult fromSerratia -free or Serratia -infected aphids was placed on a fifth trifoliate leaf (counting from the base) of 4-week-old M. truncatula to reproduce. Newborns were counted and moved to another $M$. truncatula plant. Fifty newborn nymphs were individually recorded every 7 to 9 hours for their developmental status. Data were collected from 50 aphids for both aphid populations.

Quantitative PCR. For gene expression analyses of plants and aphids, total RNA of each sample was isolated from $100 \mathrm{mg}$ leaves of plant fed by aphid for $24 \mathrm{~h}$ using the RNA Easy Mini Kit (Qiagen, Valencia, CA, USA), 5 pea aphids using TRNzol A+ (Tiangen, Beijing, China) or 20 salivary glands using the Absolutely RNA Nanoprep Kit (Agilent Technologies Inc., Santa Clara, United States), respectively. RNA (1 $\mu$ g) was used to synthesize cDNAs $(20 \mu l)$ with FastQuant RT Kit (Tiangen, Beijing, China) for each sample. To detect gene expression, qPCR was performed in a $20 \mu \mathrm{L}$ reaction volume with $2 \times$ SYBR Premix EX TaqTM (Qiagen, USA) master mix using gene-specific primers (Table S4) for phenylalanine ammonia lyase $(P A L)$, nonexpresser of PR genes 1 (NPR1), pathogenesis-related protein 1 (PR1), lipoxygenase 2 (LOX2), allene oxide synthase 2 (AOS2) in plants and $A p H R C$ in aphids and the salivary glands. Reactions were carried out on the Mx 3500P detection system (Stratagene, La Jolla, CA, USA): 3 min at $95{ }^{\circ} \mathrm{C}$; followed by 40 cycles of $30 \mathrm{~s}$ at $95{ }^{\circ} \mathrm{C}, 30 \mathrm{~s}$ at $56{ }^{\circ} \mathrm{C}$ and $30 \mathrm{~s}$ at $72{ }^{\circ} \mathrm{C}$, and finally stay at $72{ }^{\circ} \mathrm{C}$ for $3 \mathrm{~min}$. Six biologically replicates were conducted for each treatment and each biological replicate contained three technical repeats.

Measurement of ROS Production. ROS production in M. truncatula leaves was monitored using 2'7'dichlorofluorescin diacetate (DCFH-DA) (Beyotime Institute of Biotechnology, China). Its oxidation by ROS generates fluorescent 2',7'-dichlorofluorescein (DCF). Briefly, adaxial leaves were shaved to remove trichome. They were then soaked in either $1 \mathrm{ml}$ aphid saliva or $1 \mathrm{ml} 15 \%$ source solution for $6 \mathrm{~h}$ in dark, followed by infiltration with $10 \mu \mathrm{M}$ of DCFH-DA in phosphate buffer (PBS, $\mathrm{pH} 7.4$ ) for $90 \mathrm{~s}$ in $5 \mathrm{ml}$ syringes, and 3 washes with phosphate buffer. These leaves then placed in dark for $20 \mathrm{~min}$ before fluorescence detection with a Zeiss LSM710 laser confocal microscope (Zeiss, Germany). Leaf cuttings were sandwiched between two microscope coverslips with the adaxial side facing the 488-nm argon laser. Once excited, the fluorescence emissions were detected through wavelength bands $510-550 \mathrm{~nm}$ for dichlorofluorescein and 650-750 nm for the red fluorescence for chloroplasts (Lei et al., 2016). Images were analyzed using imaging system software (Zen, Zeiss, Germany).

Expression of $\boldsymbol{A p H R C}$ in M. truncatula. The full-length cDNA of $A p H R C$ was amplified and ligated into a binary vector $\mathrm{pBWA}(\mathrm{V}) \mathrm{HS}$. The construct was sequence-validated and transformed into Agrobacterium tumefaciens strain GV3101. When recombinant Agrobateria were grown in liquid LB medium (10 g/L tryptone, $5 \mathrm{~g} / \mathrm{L}$ yeast extract, $10 \mathrm{~g} / \mathrm{L} \mathrm{NaCl}, 50 \mu \mathrm{g} / \mathrm{ml}$ kanamycin) at $28{ }^{\circ} \mathrm{C}$ to an $\mathrm{OD}_{600}$ of 1 , cells were collected by centrifugation and resuspended in infiltration buffer (10 mM MES, $10 \mathrm{mM} \mathrm{MgCl} 2,200 \mu \mathrm{M}$ acetosyringone, pH5.7). Leaves of 4 week-old $M$. truncatula were infiltrated with A. tumefaciens . The uninfiltrated leaf area was harvested after $12 \mathrm{~h}$ for RNA isolation and cDNA synthesis. The cDNA samples were subjected to qPCR to detect $A p H R C$ expression. Aphid feeding behavior, expression of JA- and SA-pathway genes and $\mathrm{H}_{2} \mathrm{O}_{2}$ production were recorded $12 \mathrm{~h}$ after infiltration.

Fluorescent Signal Analysis. TIFF files were imported into Fiji (Image J) v1.52n (National Institutes of Health, USA). Fluorescence values were analyzed by GFP fluorescence of every photo as a whole. $\Delta \mathrm{F} / \mathrm{F}_{0}$ was calculated according to the equation $\Delta \mathrm{F} / \mathrm{F}_{0}=\left(\mathrm{F}_{\mathrm{t}}-\mathrm{F}_{0}\right) / \mathrm{F}_{0}$, where $\mathrm{F}_{0}$ was the first fluorescence value of the series photos and $\mathrm{F}_{\mathrm{t}}$ was the current fluorescence value.

\section{Results}

S. symbiotica Improves Aphid Feeding . To determine the effect of S. symbiotica on aphid feeding activity, we established Serratia -rebuilt aphids by injecting the hemolymph ofSerratia -infected aphids into Serratia -free aphids to exclude antibiotic influence. Electrical penetration graph (EPG) was used to monitor the feeding behavior of pea aphid on M. truncatula. Serratia -free aphids spent more time secreting saliva 
into sieve elements phase (E1 wave) and less time ingesting phloem (E2 wave) than Serratia -infected andSerratia -rebuilt aphids (Figure 1a and b). TheSerratia -rebuilt aphids began the first phloem ingestion phase significantly earlier than Serratia -free aphids although such difference was not statistically significant betweenSerratia -infected and Serratia -free aphids (Figure 1c). Shorter salivary secretion and longer passive feeding suggested that harboring S. symbiotica promoted feeding efficiency of pea aphids (Table S1).

Localization of S. symbiotica and B. aphidicola in Aphids. Fluorescence in situ hybridization (FISH) was used to determine the locations of S. symbiotica and B. aphidicolain pea aphids. As expected, only $B$. aphidicola was detected in bacteriocytes of Serratia -free aphids (Figure 1d), WhereasS. symbiotica was also seen near bacteriocytes inSerratia -infected and rebuilt aphids (Figure 1e and f).

Serratia-infected Aphids Avoided Triggering Strong Plant Defense. To determine the effect of Serratia -infection on aphid feeding-induced plant defense, we analyzed defense gene expression in $M$. truncatula infested by Serratia -free vs.Serratia -infected aphids. Compared with Serratia -free aphids, Serratia -infected aphids triggered lower gene expression levels of $P A L, N P R 1, P R 1$ in the SA pathway, as well as AOS2 and LOX2 in the JA pathway (Figure 2a-e). Consistently, the EPG experiment indicated that Serratia -infected aphids had longer feeding duration than Serratia -free aphids.

We then assessed the ROS levels in plants after aphid infestation or saliva infiltration. Upon $6 \mathrm{~h}$ infestation, Serratia -infected aphids triggered less $\mathrm{H}_{2} \mathrm{O}_{2}$ accumulation in plant leaves than Serratia -free aphids (Figure 2f). Furthermore, infiltration of saliva from Serratia -free aphids for $6 \mathrm{~h}$ led to a stronger fluorescence than from Serratia -infected aphid as well as mock-infiltration (Figure 2g). Presumably,Serratia -infected aphids would suffer less from ROS defense thanSerratia -free aphids, in agreement with the observation thatSerratia -infected aphids spent less time probing relative toSerratia -free aphids

Since $\mathrm{Ca}^{2+}$ is a ubiquitous signal that activates plant defense, we further studied the effect of aphid saliva on the cytosolic $\mathrm{Ca}^{2+}$ dynamics in plants using CaMV35S: GCaMP3 transgenic N. benthamiana. Saliva collected fromSerratia -free and Serratia -infected aphids both induced a robust $\mathrm{Ca}^{2+}$ signal within the initial $90 \mathrm{~s}$ in a $300 \mathrm{~s}$ time course whereas $15 \%$ sucrose control did not (Figure 2h). After $90 \mathrm{~s}$ however, $\mathrm{Ca}^{2+}$ signal decreased substantially in plants infiltrated with saliva from Serratia -infected aphids but remained high in plants infiltrated with saliva fromSerratia -free aphids for the remaining time period (Figure 2I; Movie S1-S3). Apparently, saliva of Serratia -infected aphids significantly suppressed $\mathrm{Ca}^{2+}$ signal.

S. symbiotica Modulates Gene Expression in Aphid Salivary Glands. To assess the effect of $S$. symbiotica infection on the gene transcription of salivary glands of pea aphids, the transcriptomic analyses were conducted using salivary glands of Serratia -free and Serratia -infected aphids. Of the 18,598 annotated genes, $S$. symbiotica significantly down-regulated the expression of 373 genes and up-regulated 347 genes (Figure 3a; Table S7, S8). Among the differentially expressed genes, 17 up-regulated and 37 down-regulated genes were predicted to contain signal peptides (Table S5 and S6). A gene annotated with ApHRC was almost 10 folds higher in salivary glands of Serratia -infected aphids than that of Serratia -free aphids (Figure $3 \mathrm{~b}$ ). The $2.3 \mathrm{kbp} A p H R C$ encodes a 448 amino acid protein with a predicted signal peptide and two putative $\mathrm{Ca}^{2+}$-binding domains (Figure $\mathrm{S} 2$ ).

$A p H R C$ expressed preferentially in a symmetrically disposed pairs of large secretory cells of the middle-lower cells of principal salivary glands (Figure 3c). Increase expression in the salivary glands and in the aphid body was detected in the presence of $S$. symbiotica (Figure 3d and e).

$A p H R C$ Facilitated Feeding of Serratia-infected Aphids. To investigate whether ApHRC influenced the feeding ofSerratia -infected aphid, RNAi was performed to silence $A p H R C$. Twenty-four $\mathrm{h}$ after ds $H R C$ -RNA injection, the $A p H R C$ expression level decreased $53 \% \pm 12 \%$ in the body and $95 \% \pm 4 \%$ in salivary glands (Figure $4 \mathrm{a}$ and b). ApHRC -silencedSerratia -infected aphids displayed prolonged salivary secretion (E1 wave), decreased the phloem ingestion phase (E2 wave) (Figure 4c and d), and increased intracellular punctures (pd wave, i.e. more navigation time to the phloem) (Figure 4e), which inevitably led to reduced feeding efficiency (Table S2). 
$A p H R C$ Suppresses Plant Defense by Eliminating the $\mathbf{C a}^{2+}$ Elevation. Silencing $A p H R C$ inSerratia -infected aphids resulted in higher PAL and lower $L O X 2$ expression, but no change in PR1,NPR1 and AOS2 (Figure 4f-j). Furthermore, after $6 \mathrm{~h}$ infestation, $A p H R C$ - silenced aphid produced significantly more $\mathrm{H}_{2} \mathrm{O}_{2}$ and stronger green fluorescence than the ds GFP control aphids (Figure 4k and l), suggesting that ApHRC can efficiently suppress plant ROS signals. The $\mathrm{Ca}^{2+}$ dynamics resembled that of Serratia -free aphids (Figure $4 \mathrm{~m}$ and $\mathrm{n}$; Movie S4-S6). These results indicated $A p H R C$ was able to suppress the plant $\mathrm{Ca}^{2+}$ signal during aphid infestation.

Expression of $A p H R C$ in Plants Facilitated Feeding of $A p H R C$-silenced Serratia-infected Aphids . To further determine the effects of overexpression of $A p H R C$ on plant defense and the feeding activity of $A p H R C$ - silenced Serratia -infected aphids, we transiently expressed the full-length of ApHRC in $M$. truncatula by agroinfiltration. Overexpression of $A p H R C$ in $M$. truncatula shortened salivary secretion (E1 wave) and elongated the duration of phloem feeding (E2 wave) (Figure 5a and b; Table S3). Induced $P A L$ and suppressed LOX2 expression was also observed (Figure 5c-g). While $\mathrm{H}_{2} \mathrm{O}_{2}$ was abundant in the plant cells in the vector-infiltrated plants, the $A p H R C$ infiltrated plants barely accumulated any (Figure $5 \mathrm{~h}$ and i). Therefore, the transient expression of $A p H R C$ in M. truncatula leaves led to the inhibition of plant defense responses.

The Infection of S. symbiotica Improves the development of Pea Aphids. To determine the effect of $S$. symbioticainfection on the performance of pea aphids, offspring number, mean relative growth rate (MRGR) and the developmental duration of nymphs were compared between Serratia -free aphids andSerratia -infected aphids when reared on $M$. truncatula .S. symbiotica infection did not significantly affect MRGR and offspring number of pea aphids (Figure 6a and b). By contrast,Serratia -infected aphids had significantly shorter $2^{\text {nd }}$ instar and $3^{\text {rd }}$ instar duration than those of Serratia -free aphids (Figure 6c). Therefore,S. symbiotica infection improved aphid development.

\section{Discussion}

The beneficial effects of microbial facultative endosymbionts on aphid hosts are well recognized, especially when aphids are challenged by biotic and abiotic stresses. On the other hand, these endosymbionts usually impair aphid growth and development, or suppress reproduction under normal environment as physiological costs (Chen, Montllor, \& Purcell, 2000; Ferrari, Darby, Daniell, Godfray, \& Douglas, 2004; Oliver, Moran, \& Hunter, 2005). Facultative endosymbionts in pea aphids including H. defensa, Spiroplasma, Rickettsiadecrease longevity, fecundity and/or body weight of the aphid (Fukatsu, Tsuchida, Nikoh, \& Koga, 2001; Polin, Simon, \& Outreman, 2014; Russell, \& Moran, 2006). By contrast, Serratia -infected aphids developed more rapidly than Serratia -free aphids on M. truncatula. Similarly, S. symbiotica accelerated cedar aphid Cinara cedri growth as well by directly providing essential amino acids to host, gradually turning into an obligate endosymbiont (Lamelas et al., 2011). However, few amino acid metabolic genes have been annotated in the genome of $S$. symbiotica in pea aphids to supply nutrition (Manzanomarin, Lamelas, Moya, \& Latorre, 2012). Since the field population of pea aphids had a relatively high infectious rate of S. symbiotica , we speculated that $S$. symbiotica was likely involved in the early aphid feeding and colonization on $\mathrm{Me}$ dicagoplants by supplying sufficient nutrition leading to accelerated population growth (Figure S1). In the current study, we demonstrated that $S$. symbiotica sharply up-regulated $A p H R C$ in salivary glands of pea aphids, which efficiently suppressed $\mathrm{Ca}^{2+}$ signal and plant defense.

S. symbiotica is usually localized freely within hemolymph or near the bacteriocytes within pea aphids (Moran, Russell, Koga, \& Fukatsu, 2005). S. symbiotica infection could impair the cellular immunity of pea aphid and decreased indole-3-lactate, an antioxidant, which may down-regulate ROS in aphid hemolymph (Burke, Fiehn, \& Moran, 2010). Also, genome sequencing revealed that the IMD pathway along with many immune genes such as those encoding peptidoglycan recognition proteins and AMPs are lost in the pea aphid, possibly facilitating association of aphids with microbial symbionts (Gerardo et al., 2010). Interestingly, $S$. symbiotica increased drastically the expression of $A p H R C$, a gene encoding a presumably secretory protein in salivary glands (Figure 3B). Although little is known concerning its function in aphid immune response to the infection of $S$. symbiotica, we suspected that up-regulation of $A p H R C$ possibly suppressed immune 
responses of aphid during the infection of $S$. symbiotica via quenching the early elevation of the $\mathrm{Ca}^{2+}$ signal as well.

Wounding caused by aphid feeding elicits plant $\mathrm{Ca}^{2+}$ influx, a signal that turns on down-stream defenses, such as sieve element occlusion and ROS accumulation to prevent aphid feeding (Sun, Voorrips, Kaauwen, Visser, \& Vosman, 2020). However, aphids secret salivary effectors to maintain constant phloem feeding (Mutti et al., 2008). Notably, several effectors have calcium-binding domains, such as Armet, that suppresses plant $\mathrm{Ca}^{2+}$ signal to facilitate aphid feeding (Cui et al., 2019). Here, Serratia infection aphids, either infested by aphids or infiltrated with saliva, led to weaker ROS and less $\mathrm{H}_{2} \mathrm{O}_{2}$ concentration in plant leaves, possibly attributing to the inhibition of $\mathrm{Ca}^{2+}$ signal via $\mathrm{Ca}^{2+}$-binding inM. truncatula (Tian et al., 2019). Consistent with this notion, silencing $A p H R C$ significantly decreased the phloem ingestion time of aphids and induced higher ROS in M. truncatula, suggesting that $A p H R C$ was a Serratia -induced salivary effector that improved aphid feeding. In addition to ROS, JA and SA signaling pathways have been widely reported to be involved in plant resistance against aphids (Mohase \& Der Westhuizen, 2002; Selig, Keough, Nalam, \& Nachappa, 2016; Sun et al., 2020). Since silencing $A p H R C$ in the salivary glands or expression of $A p H R C$ in plants only affected $L O X 2$ and $P A L$ but was not significant for AOS2, NPR1 and PR1, we conclude that JA and SA signaling pathways are only weakly affected by ApHRC. By contrast, the infestation of Serratia -infected aphids triggered weaker JA and SA signaling pathways than Serratia -free aphid in terms of all measured marker genes, indicating that some other salivary effectors ofSerratia-infected aphids down-regulated the JA and SA signaling pathways instead of $A p H R C$. Since a number of differentially expressed genes were affected by Serratia infection in our transcriptomic data of salivary glands, their function in suppression of plant defenses may be responsible for the discrepancy betweenSerratia -infection and ApHRC effects in activation of JA and SA signaling pathways. For example, Serratia -infected aphid could decrease the expression of a chemosensory protein (CSP) MP10 known to up-regulate JA and SA pathways of the host plant (Rodriguez, Stam, Warbroek, \& Bos, 2014). Furthermore, HRC was also annotated in several other aphid species including Myzus persicae ,Diuraphis noxia, and Rhopalosiphum maidis, suggesting that the function of $H R C$ might be conserved in aphids (Figure S3). In addition, a recent study showed that the mutation of a $H R C$ in wheat enhanced the resistance against Fusarium head blight (Li et al., 2019). Most likely, $H R C$ could down-regulate the $\mathrm{Ca}^{2+}$ responses to fungi infection, conferring susceptibility to the fungal diseases in wheat. Suppressing plant defense, as a newly discovered function of S. symbiotica, may explain its high prevalence in our field sampling compared with other facultative symbionts (Figure S1).

Growing evidence suggests that microbial mutualistic symbioses of insects could be orally secreted into a plant and directly manipulate the plant defenses (Chung, Rosa, Hoover, Luthe, \& Felton, 2013). For example, a psyllid bacterial endosymbiont Candidatus Liberibacter psyllaurous could suppress JA and SA defensive signaling pathways of tomato plants (Casteel, Hansen, Walling, \& Paine, 2012). The Colorado potato beetle secretes symbiotic bacteria to elicit SA-regulated defense while suppressing the efficient JA signaling pathway (Sorokan, Burkhanova, Benkovskaya, \& Maksimov, 2019). Since most of the symbionts reside in bacteriocytes, gut, and hemolymph of insects, it is more common that the endosymbionts modulate the insect-plant interaction by indirectly regulating the transcripts of insect salivary gland genes rather than directly being secreted into the plant. We have shown that, on the basis of the transcriptome of salivary glands, the infection ofS. symbiotica could modify a number of salivary proteins of pea aphids (Table S5-S8). Our study has revealed a novel strategy employed by aphids where they host microbial facultative symbionts to benefit their own feeding. Serratia -infected aphids are thus more likely to be successful in host colonization and population expansion onMedicago plants.

\section{Author Contributions}

F.G. and Y.S. conceived the original screening and research plans; H.G. and Y.S. supervised the experiments; Q.W. and X.L. performed most of the experiments; Q.W., E.Y. conducted the field sampling; Q.W. and H.G. designed the experiments and analyzed the data; Q.W., Y.S. and K.Z-S. wrote the article with contributions of all the authors.

\section{Acknowledgments}


We thank Professor Zi-Hua Zhao for conducted field sampling, and Dr. Jing Gao for sharing RT-qPCR laboratory techniques. This project was supported by the National key R\&D Program of China (no. 2017YFD0200400) and the Strategic Priority Research Program of the Chinese Academy of Sciences (no. XDB11050400) and the National Nature Science Fund of China (nos. 31770452 and 31870394).

\section{Declaration of Interests:}

The authors declare no competing interests.

\section{References}

Aidemark, M., Andersson, C., Rasmusson, A. G., \& Widell, S. (2009). Regulation of callose synthase activity in situ in alamethicin-permeabilized Arabidopsis and tobacco suspension cells. BMC Plant Biology , 9(1), 2727.

Burke, G. R., Fiehn, O., \& Moran, N. A. (2010). Effects of facultative symbionts and heat stress on the metabolome of pea aphids. The ISME Journal , 4(2), 242-252.

Casteel, C. L., Hansen, A. K., Walling, L. L., \& Paine, T. D. (2012). Manipulation of plant defense responses by the tomato psyllid (Bactericerca cockerelli) and its associated endosymbiont Candidatus Liberibacter psyllaurous. PLOS ONE, 7(4).

Chung, S. H., Rosa, C., Hoover, K., Luthe, D. S., \& Felton, G. W. (2013). Colorado potato beetle manipulates plant defenses in local and systemic leaves. Plant Signaling 83 Behavior , 8(12).

Chaudhary, R., Atamian, H. S., Shen, Z., Briggs, S. P., \& Kaloshian, I. (2014). GroEL from the endosymbiont Buchnera aphidicola betrays the aphid by triggering plant defense. Proceedings of the National Academy of Sciences of the United States of America , 111(24), 8919-8924.

Chen, D., Montllor, C. B., \& Purcell, A. H. (2000). Fitness effects of two facultative endosymbiotic bacteria on the pea aphid, Acyrthosiphon pisum, and the blue alfalfa aphid, A. kondoi . Entomologia Experimentalis Et Applicata , 95(3), 315-323.

Costopoulos, K., Kovacs, J. L., Kamins, A., \& Gerardo, N. M. (2014). Aphid facultative symbionts reduce survival of the predatory lady beetle Hippodamia convergens . BMC Ecology, 14(1), 5-5.

Cui, N., Lu, H., Wang, T., Zhang, W., Kang, L., \& Cui, F. (2019). Armet, an aphid effector protein, induces pathogen resistance in plants by promoting the accumulation of salicylic acid. Philosophical Transactions of the Royal Society B , 374(1767).

Defalco, T. A., Toyota, M., Phan, V., Karia, P., Moeder, W., Gilroy, S., \& Yoshioka, K. (2017). Using GCaMP3 to Study $\mathrm{Ca}^{2+}$ Signaling in NicotianaSpecies. Plant and Cell Physiology, 58(7), 1173-1184.

De Vos, M., Van Oosten, V. R., Van Poecke, R. M., Van Pelt, J. A., Pozo, M. J., Mueller, M. J., ... \& Pieterse, C. M. (2005). Signal signature and transcriptome changes of Arabidopsis during pathogen and insect attack. Molecular Plant-microbe Interactions, 18(9), 923-937.

Douglas, A. E., \& Prosser, W. A. (1992). Synthesis of the essential amino acid tryptophan in the pea aphid (Acyrthosiphon pisum ) symbiosis. Journal of Insect Physiology , 38(8), 565-568.

Douglas, A. E. (1998). Nutritional Interactions in Insect-Microbial Symbioses: Aphids and Their Symbiotic Bacteria Buchnera . Annual Review of Entomology , 43(1), 17-37.

Doremus, M. R., \& Oliver, K. M. (2017). Aphid Heritable Symbiont Exploits Defensive Mutualism. Applied and Environmental Microbiology, 83(8).

Farmer, E. E., Gasperini, D., \& Acosta, I. F. (2014). The squeeze cell hypothesis for the activation of jasmonate synthesis in response to wounding. New Phytologist, 204(2), 282-288. 
Ferrari, J., Darby, A. C., Daniell, T. J., Godfray, H. C., \& Douglas, A. E. (2004). Linking the bacterial community in pea aphids with host-plant use and natural enemy resistance. Ecological Entomology , 29(1), $60-65$.

Fukatsu, T., Tsuchida, T., Nikoh, N., \& Koga, R. (2001). Spiroplasma symbiont of the pea aphid,Acyrthosiphon pisum (Insecta : Homoptera). Applied andEnvironmental Microbiology, 67(3), 1284-1291.

Frantz, A., Calcagno, V., Mieuzet, L., Plantegenest, M., \& Simon, J. (2009). Complex trait differentiation between host-populations of the pea aphid Acyrthosiphon pisum (Harris): implications for the evolution of ecological specialisation. Biological Journal of The Linnean Society , 97(4), 718-727.

Gerardo, N. M., Altincicek, B., Anselme, C., Atamian, H. S., Barribeau, S. M., De Vos, M., .. \& Vilcinskas, A. (2010). Immunity and other defenses in pea aphids, Acyrthosiphon pisum . Genome Biology , 11(2), 1-17.

Henry, L. M., Peccoud, J., Simon, J., Hadfield, J. D., Maiden, M., Ferrari, J., \& Godfray, H. C. (2013). Horizontally Transmitted Symbionts and Host Colonization of Ecological Niches. Current Biology , 23(17), 1713-1717.

Hogenhout, S. A., \& Bos, J. I. (2011). Effector proteins that modulate plant-insect interactions. Current Opinion in Plant Biology, 14(4), 422-428.

Hopper, K. R., Kuhn, K. L., Lanier, K., Rhoades, J. H., Oliver, K. M., White, J. A., .. \& Heimpel, G. E. (2018). The defensive aphid symbiont Hamiltonella defensa affects host quality differently for Aphelinus glycinis versus Aphelinus atriplicis . Biological Control , 116, 3-9.

Jaouannet, M. L., Rodriguez, P. A., Thorpe, P., Lenoir, C. J., Macleod, R., Escuderomartinez, C., \& Bos, J. I. (2014). Plant immunity in plant-aphid interactions. Frontiers in Plant Science ,, 663-663.

Laitinen, T., Morreel, K., Delhomme, N., Gauthier, A., Schiffthaler, B., Nickolov, K., .. \& Karkonen, A. (2017). A Key Role for Apoplastic $\mathrm{H}_{2} \mathrm{O}_{2}$ in Norway Spruce Phenolic Metabolism. Plant Physiology , 174(3), 1449-1475.

La Pena, E. D., Vandomme, V., \& Frago, E. (2014). Facultative endosymbionts of aphid populations from coastal dunes of the North Sea. Belgian Journal of Zoology , 144(1), 41-50.

Lamelas, A., Gosalbes, M. J., Manzanomarin, A., Pereto, J., Moya, A., \& Latorre, A. (2011). Serratia symbiotica from the AphidCinara cedri : A Missing Link from Facultative to Obligate Insect Endosymbiont. PLOS Genetics , 7(11).

Leather S.R. \& Dixon A.F.G (1984) Aphid growth and reproductive rates. Entomologia Experimentalis et Applicata , 35, 137-140.

Lei R, Du Z, Qiu Y, Zhu S. (2016). The detection of hydrogen peroxide involved in plant virus infection by fluorescence spectroscopy.Luminescence , 31, 1158-1165.

Leonardo, T. E., \& Muiru, G. T. (2003). Facultative symbionts are associated with host plant specialization in pea aphid populations. PROCEEDING OF THE ROYAL SOCIETY B , 270, S209-S212.

Li, Z., Feng, Z., Maeli, M., Jian, Y., Sheng, Y. H. (2017). Jasmonate signaling and manipulation by pathogens and insects. Journal of experimental botany , 68(6), 1371-1385.

Li, G., Zhou, J., Jia, H., Gao, Z., Fan, M., Luo, Y., .. \& \& Ma, Z. (2019). Mutation of a histidine-rich calcium-binding-protein gene in wheat confers resistance to Fusarium head blight. Nature Genetics , 51(7), 1106-1112.

Łukasik, P., Van Asch, M., Guo, H., Ferrari, J., \& Godfray, H. C. (2013). Unrelated facultative endosymbionts protect aphids against a fungal pathogen. Ecology Letters , 16(2), 214-218.

Manzanomarin, A., Lamelas, A., Moya, A., \& Latorre, A. (2012). Comparative Genomics of Serratiaspp.: Two Paths towards Endosymbiotic Life. PLOS ONE , 7(10). 
Mewis, I., Tokuhisa, J. G., Schultz, J. C., Appel, H. M., Ulrichs, C., \& Gershenzon, J. (2006). Gene expression and glucosinolate accumulation in Arabidopsis thaliana in response to generalist and specialist herbivores of different feeding guilds and the role of defense signaling pathways. Phytochemistry , 67(22), $2450-2462$.

Mohase, L., \& Der Westhuizen, A. J. (2002). Salicylic acid is involved in resistance responses in the Russian wheat aphid-wheat interaction. Journal of Plant Physiology , 159(6), 585-590.

Montllor C, Maxmen A, \& Purcell AH. (2002). Facultative bacterial endosymbionts benefit pea aphids Acyrthosiphon pisum under heat stress. Ecol Entomol, 27: 189-195.

Moran, N. A., Russell, J. A., Koga, R., \& Fukatsu, T. (2005). Evolutionary Relationships of Three New Species of Enterobacteriaceae Living as Symbionts of Aphids and Other Insects. Applied and Environmental Microbiology , 71(6), 3302-3310.

Moran, P. J., \& Thompson, G. A. (2001). Molecular Responses to Aphid Feeding in Arabidopsis in Relation to Plant Defense Pathways. Plant Physiology, 125(2), 1074-1085.

Moran, P. J., Cheng, Y., Cassell, J. L., \& Thompson, G. A. (2002). Gene Expression Profiling of Arabidopsis thaliana in Compatible Plant-Aphid Interactions. Archives of Insect Biochemistry and Physiology , 51(4), 182-203.

Mutti, N. S., Louis, J., Pappan, L. K., Pappan, K. L., Begum, K., Chen, M., .. \& Reeck, G. R. (2008). A protein from the salivary glands of the pea aphid, Acyrthosiphon pisum, is essential in feeding on a host plant. Proceedings of the National Academy of Sciences of the United States of America, 105(29), 9965-9969.

Oliver, K. M., Russell, J. A., Moran, N. A., \& Hunter, M. S. (2003). Facultative bacterial symbionts in aphids confer resistance to parasitic wasps. Proceedings of the National Academy of Sciences of the United States of America, 100(4), 1803-1807.

Oliver, K. M., Moran, N. A., \& Hunter, M. S. (2005). Variation in resistance to parasitism in aphids is due to symbionts not host genotype. Proceedings of the National Academy of Sciences of the United States of America , 102(36), 12795-12800.

Pegadaraju, V., Knepper, C., Reese, J. C., \& Shah, J. (2005). Premature Leaf Senescence Modulated by the Arabidopsis PHYTOALEXIN DEFICIENT4 Gene Is Associated with Defense against the Phloem-Feeding Green Peach Aphid. Plant Physiology, 139(4), 1927-1934.

Polin, S., Simon, J., \& Outreman, Y. (2014). An ecological cost associated with protective symbionts of aphids. Ecology and Evolution , 4(6), 836-840.

Rodriguez, P. A., Stam, R., Warbroek, T., \& Bos, J. I. (2014). Mp10 and Mp42 from the aphid speciesMyzus persicae trigger plant defenses in Nicotiana benthamiana through different activities. Molecular Plant-microbe Interactions , 27(1), 30-39.

Russell, J. A., \& Moran, N. A. (2006). Costs and benefits of symbiont infection in aphids: variation among symbionts and across temperatures. Proceedings of the Royal Society B , 273, 603-610.

Selig, P., Keough, S., Nalam, V. J., \& Nachappa, P. (2016). Jasmonate-dependent plant defenses mediate soybean thrips and soybean aphid performance on soybean. Arthropod-plant Interactions , 10(4), 273-282.

Simon, J. C., Carre, S., Boutin, M., Leterme, N. P., Munoz, B. S., Latorre, A., \& Bournoville, R. (2003). Host-based divergence in populations of the pea aphid: insights from nuclear markers and the prevalence of facultative symbionts. Proceedings of the Royal Society B , 270, 1703-1712.

Sorokan, A. V., Burkhanova, G. F., Benkovskaya, G. V., \& Maksimov, I. V. (2019). Colorado potato beetle microsymbiont Enterobacter BC-8 inhibits defense mechanisms of potato plants using crosstalk between jasmonate- and salicylate-mediated signaling pathways. Arthropod-plant Interactions , https://doi.org/10.1007/s11829019-09732-w. 
Sun, M., Voorrips, R. E., Kaauwen, M., Visser, R., \& Vosman, B. (2020). The ability to manipulate ROS metabolism in pepper may affect aphid virulence. Horticulture Research , 7, 6 .

Tian, W., Hou, C., Ren, Z., Wang, C., Zhao, F., Dahlbeck, D., .. \& \& Luan, S. (2019). A calmodulin-gated calcium channel links pathogen patterns to plant immunity. Nature, 572(7767), 131-135.

Tjallingii, W. F., \& Esch, T. H. (1993) Fine structure of the stylet route in plant tissues by some aphids. Physiol. Entomol. , 18, 317-328.

Vincent, T. R., Avramova, M., Canham, J., Higgins, P., Bilkey, N., Mugford, S.T., .. Sanders, D. (2017). Interplay of Plasma Membrane and Vacuolar Ion Channels, Together with BAK1, Elicits Rapid Cytosolic Calcium Elevations inArabidopsis during Aphid Feeding. The Plant Cell , 29(6), 1460-1479.

Wang, W., Dai, H., Zhang, Y., Chandrasekar, R., Luo, L., Hiromasa, Y., . . \& Cui, F. (2015). Armet is an effector protein mediating aphid-plant interactions. The FASEB Journal , 29(5), 2032-2045.

Fig 1. S. symbiotica facilitated pea aphids' feeding on M. truncatula (cv. A17) and its location in aphid body.

(a-c) EPG results of Serratia -: Serratia -free,Serratia +: Serratia -infected, and Serratia +r:Serratia rebuilt aphids for $8 \mathrm{~h}$ on $M$. truncatula . (a) Time spent on salivary secretion into sieve elements (E1 wave) $(\mathrm{n}=10)$. (b) Ingestion time (E2 wave) $(\mathrm{n}=10)$. (c) Time to phloem ingestion $(\mathrm{n}=10)$. (d-f) Fluorescence in situ hybridization (FISH) to detect B. aphidicola and S. symbiotica in aphid abdomen. Aphid DNA was stained with 4',6-diamidino-2-phenylindole (blue), B. aphidicolaDNA was hybridized with Cy5-labeled DNA probe (red), S. symbiotica DNA was hybridized with Cy3-labeled DNA probe (green). (d)B. aphidicola in Serratia -free aphids. (e) B. aphidicola and S. symbiotica in Serratia -infected aphids. (f) B. aphidicola and $S$. symbiotica inSerratia -rebuilt aphids. The data shown are mean \pm standard error (SEM). * indicate significant differences among different treatments at $\mathrm{P}<0.05\left({ }^{*} \mathrm{P}<0.05,{ }^{*} \mathrm{P}<0.01,{ }^{* * *} \mathrm{P}<0.001\right)$ one-way ANOVA analysis for EPG data.

Fig 2. M. truncatula (cv. A17) defense triggered by aphids' feeding or aphid saliva.

(a-e) Relative expression levels of M. truncatula JA and SA pathway genes induced by aphids. Serratia -: Serratia -free aphids and Serratia +: Serratia -infected aphid. (a) $P A L$, phenylalanine ammonia lyase; (b) NPR1, nonexpresser of PR genes 1; (c) PR1, pathogenesis-related protein 1; (d)LOX2, lipoxygenase 2; (e) AOS2 , allene oxide synthase 2. ( $\mathrm{n}=6)$. (f) $\mathrm{H}_{2} \mathrm{O}_{2}$ concentration induced by 10 Serratia -infected and Serratia -free aphids, respectively for $6 \mathrm{~h}(\mathrm{n}=6)$. (g) Subcellular localization of fluorescent probes, DCFH-DA, in leaves of M. truncatula infiltrate bySerratia -free (the middle panel) and Serratia -infected aphid saliva (the lower panel). Control (the upper panel): $15 \%$ sucrose solution treatment. Bars $=100 \mu \mathrm{m}$. (h) Normalized fluorescence $\left(\Delta \mathrm{F} / \mathrm{F}_{0}\right)$ of $\mathrm{Ca}^{2+}$ signal measurements every $3 \mathrm{~s}$ in $N$. benthamiana detached leaves infiltrated bySerratia -: Serratia -free, and Serratia +:Serratia -infected aphids saliva. Control: $15 \%$ sucrose solution treatment. $\Delta \mathrm{F}$, the difference between measured fluorescence and the fluorescence of the very first picture $\left(\mathrm{F}_{0}\right)(\mathrm{n}=15)$. Yellow shading indicates significant difference between Serratia - and Serratia + treatment $(\mathrm{P}<0.05)$. (i) Representative fluorescence microscope images showing fluorescence of $\mathrm{Ca}^{2+}$ signal in $N$. benthamiana leaves infiltrated by Serratia -free (the middle panel) andSerratia -infected aphid saliva (the lower panel). Control (the upper panel): $15 \%$ sucrose solution treatment. Bars $=100 \mu \mathrm{m}$. The data shown are mean \pm standard error $(\mathrm{SEM})$. ${ }^{*}$ above the bars indicate significant differences among different treatments at $\mathrm{P}<0.05$ (t-test, ${ }^{*} \mathrm{P}<0.05,{ }^{*} \mathrm{P}<0.01,{ }^{* * *} \mathrm{P}<0.001$ ).

Fig 3. The ApHRC expression level in salivary glands of pea aphids.

(a) Heat map of differentially expressed genes (DEGs) in the salivary gland of Serratia -free aphid (Serratia -) andSerratia -infected aphid (Serratia +). (b) The relative expression of the top 10 up-regulated candidate secretary saliva proteins in Serratia -infected salivary glands transcriptome when compared with Serratia -free aphid. Gene in the red box is $A p H R C$. Three biological replications were conducted in the RNAseq analysis. (c) FISH to detect the ApHRC expression level in aphid salivary glands. ApHRC mRNA was 
hybridized with 5- carboxyfluorescein (5-FAM) in red, nuclei were stained with 4',6-diamidino-2-phenylindole (DAPI) in blue. PG, principal gland; AG, accessory gland. Bars $=50 \mu \mathrm{m}$. (d) ApHRC relative expression level in aphid body $(\mathrm{n}=6)$. (e) $A p H R C$ relative expression level in aphid salivary gland $(\mathrm{n}=6)$. The data shown are mean \pm standard error (SEM). ${ }^{*}$ above the bars indicate significant differences among different treatments at $\mathrm{P}<0.05$ (t-test, ${ }^{*} \mathrm{P}<0.05,{ }^{* *} \mathrm{P}<0.01,{ }^{* * *} \mathrm{P}<0.001$ ).

\section{Fig 4. Stimulation of plant defense after $A p H R C$ was silenced.}

Relative $A p H R C$ expression in Serratia -infected (a) aphid body and (b) salivary glands $24 \mathrm{~h}$ after injection of ds $H R C$-RNA (n=6). (c-e) EPG results of ds GFP : Serratia -infected aphid injected ds GFP as control, and dsHRC :ApHRC -silenced Serratia -infected aphid for $8 \mathrm{~h}$ on M. truncatula. (c) Time spent on salivary secretion into sieve elements (E1 wave) $(\mathrm{n}=10)$. (d) Ingestion time (E2 wave) (n=10). (e) Time of intracellular punctures (pd wave) (n=10). (f-j) Relative expression of $M$. truncatula JA and SA pathway genes induced by ds $H R C$-and ds $G F P$-aphids infestation. (f) $P A L$, phenylalanine ammonia lyase; (g) NPR1, nonexpresser of PR genes 1; (h)PR1, pathogenesis-related protein 1; (i) LOX2, lipoxygenase 2; (j) AOS2, allene oxide synthase 2. $(\mathrm{n}=6)$. (k) $\mathrm{H}_{2} \mathrm{O}_{2}$ concentration induced by $10 \mathrm{ds} H R C$ and ds $G F P$ aphids, respectively for $6 \mathrm{~h}$ $(\mathrm{n}=6)$. (l) Subcellular localization of fluorescent probes, DCFH-DA, in leaves of $M$. truncatula infiltrate by ds GFP -aphid (the middle panel) and ds $H R C$ aphid saliva (the lower panel). Control (the upper panel): $15 \%$ sucrose solution treatment. Bars $=100 \mu \mathrm{m}$. $(\mathrm{m})$ Normalized fluorescence $\left(\Delta \mathrm{F} / \mathrm{F}_{0}\right)$ of $\mathrm{Ca}^{2+}$ signal measurements every $3 \mathrm{~s}$ in $N$. benthamiana detached leaves infiltrated by ds $G F P$-aphid and ds $H R C$-aphid saliva. Control: $15 \%$ sucrose solution treatment. $\Delta \mathrm{F}$, the difference between measured fluorescence and the fluorescence of the very first picture $\left(\mathrm{F}_{0}\right)(\mathrm{n}=15)$. Yellow shading indicates significant difference between ds GFP-aphids and ds $H R C$-aphid saliva treatment $(\mathrm{P}<0.05)$. (n) Representative fluorescence microscope images showing fluorescence of $\mathrm{Ca}^{2+}$ signal in $N$. benthamiana leaves infiltrated by dsGFP-aphid (the middle panel) and ds $H R C$-aphid saliva (the lower panel). Control (the upper panel): $15 \%$ sucrose solution treatment. Bars $=100 \mu \mathrm{m}$. The data shown are mean \pm standard error $(\mathrm{SEM}) .{ }^{*}$ above the bars indicate significant differences among different treatments at $\mathrm{P}<0.05$ (t-test, ${ }^{*} \mathrm{P}<0.05$, ${ }^{* *} \mathrm{P}<0.01,{ }^{* * *} \mathrm{P}<0.001$ ).

Fig 5. $A p H R C$ expression promoted aphid performance.

(a-b) EPG results of $A p H R C$-silenced Serratia -infected aphids for $8 \mathrm{~h}$ on EV: empty vector, and OEHRC : overexpression of ApHRC M. truncatula . (a) Time spent on salivary secretion into sieve elements (E1 wave) $(\mathrm{n}=10)$. (b) Ingestion time (E2 wave) $(\mathrm{n}=10)$. ( $\mathrm{c}-\mathrm{g})$ Relative expression levels of EV and OEHRC M. truncatula JA and SA pathway genes induced by ApHRC -silencedSerratia -infected aphids. (c) $P A L$ , phenylalanine ammonia lyase; (d) NPR1, nonexpresser of PR genes 1; (e) PR1, pathogenesis-related protein 1; (f) LOX2, lipoxygenase 2; (g)AOS2, allene oxide synthase 2. (h) $\mathrm{H}_{2} \mathrm{O}_{2}$ concentration of EV and OEHRC M. truncatula leaves induced by $10 A p H R C$-silenced Serratia -infected aphids, respectively for 6 $\mathrm{h}(\mathrm{n}=6)$. (i) Subcellular localization of fluorescent probes, DCFH-DA, in leaves of EV (the middle panel) and OE HRC (the lower panel) M. truncatula infiltrate by ApHRC -silencedSerratia -infected aphids. Control (the upper panel): $15 \%$ sucrose solution treatment. Bars $=100 \mu \mathrm{m}$. The data shown are mean \pm standard error (SEM). * above the bars indicate significant differences among different treatments at $\mathrm{P}<0.05$ (t-test, $\left.{ }^{*} \mathrm{P}<0.05,{ }^{* *} \mathrm{P}<0.01,{ }^{* * *} \mathrm{P}<0.001\right)$.

Fig 6. S. symbiotica influence on pea aphid MRGR, fecundity and development time.

(a) MRGR of Serratia -infected (Serratia + ) andSerratia -free (Serratia -) aphids (n=50). (b) Fecundity ofSerratia -infected and Serratia -free aphids (n=50). (c) Nymphal duration of Serratia -infected and Serratia -free aphids $(\mathrm{n}=50)$. Data shown are mean \pm standard error $(\mathrm{SEM}) .{ }^{*}$ above the bars indicate significant differences among different treatments at $\mathrm{P}<0.05$ (t-test, ${ }^{*} \mathrm{P}<0.05,{ }^{*} \mathrm{P}<0.01,{ }^{* * *} \mathrm{P}<0.001$ ). 
(a)
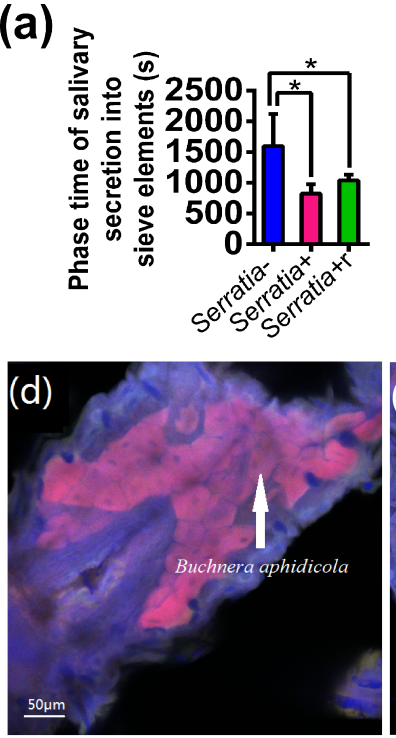

Serratia-free aphid (b)
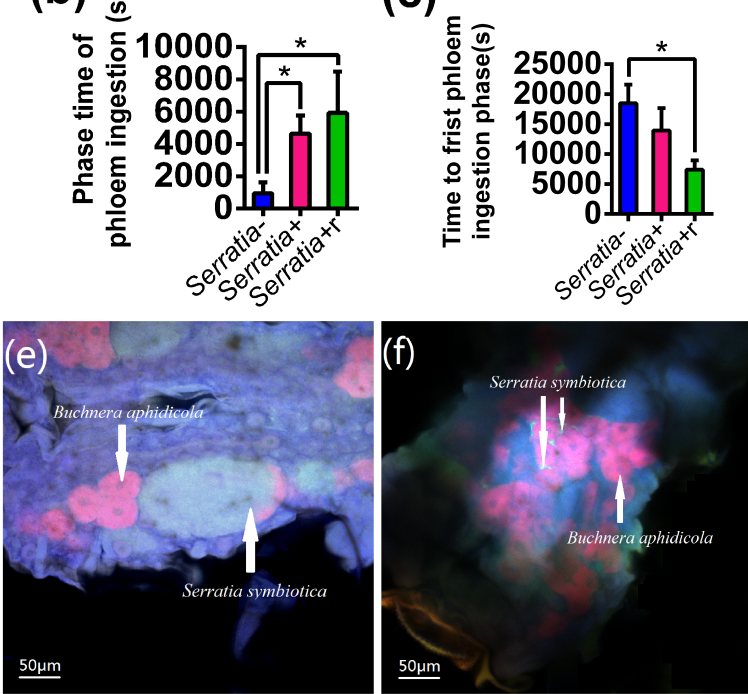

Serratia-infected aphid Serratia-rebuilt aphid 
(a)



(h)

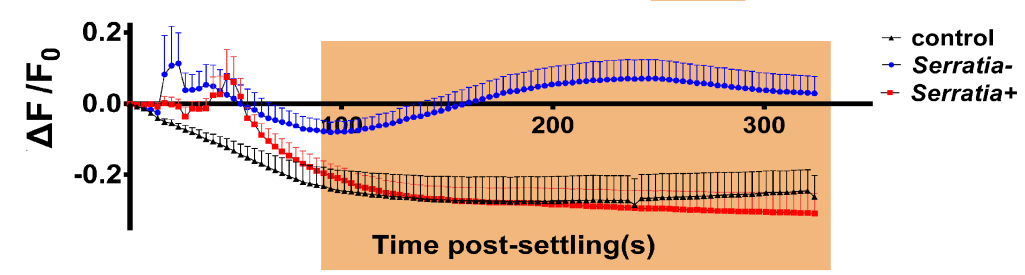

(i)

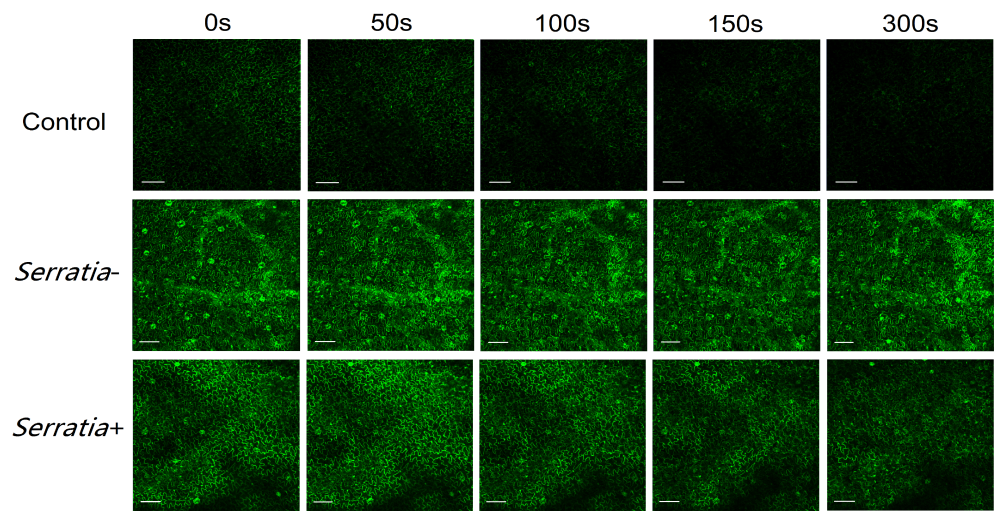



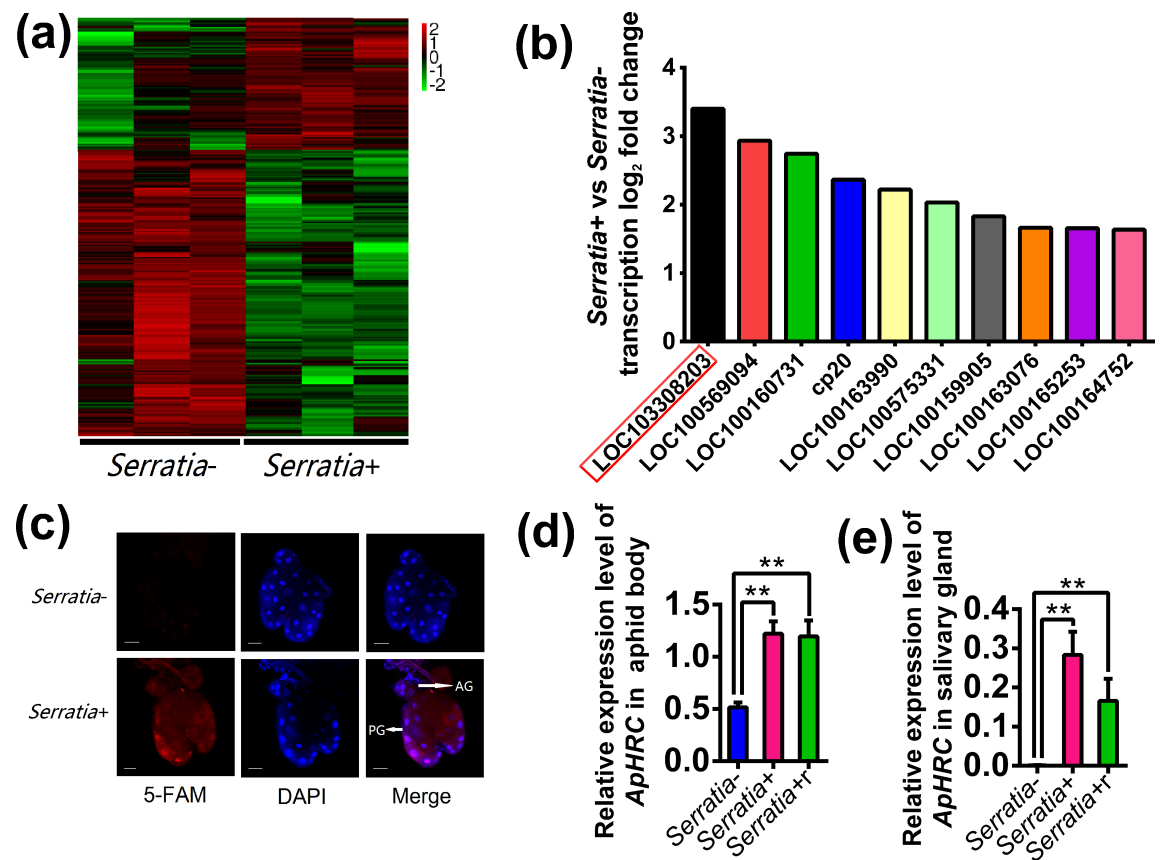

(e)

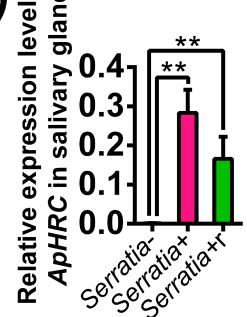




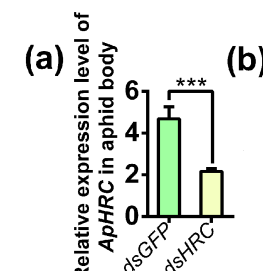

(b) $\frac{\overline{0}}{\mathrm{~s}}$ 를

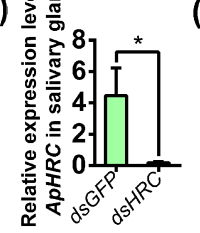

(c)

(d)
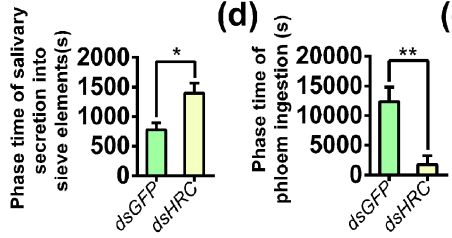

(e) $\frac{\bar{\sigma}}{\frac{\pi}{6}}$

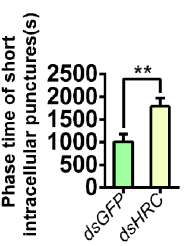

(f) $\frac{5}{0}$

PAL (g)

PR1 (h)


(i)

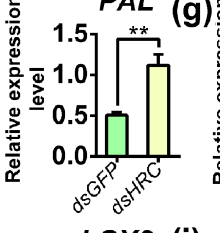

AOS2 (k)
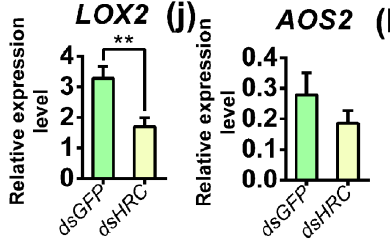

(k) $\mathrm{H}_{2} \mathrm{O}_{2}$

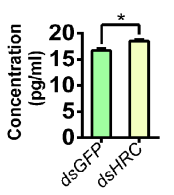

(I)
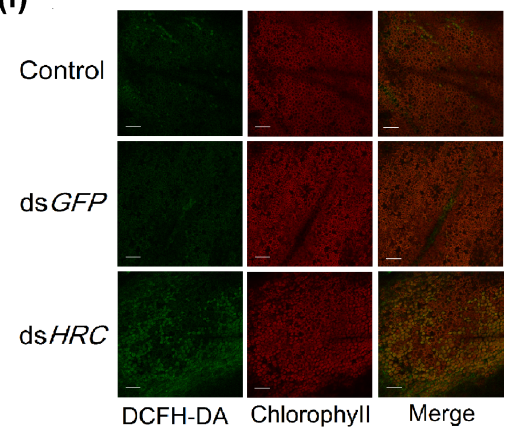

(m)
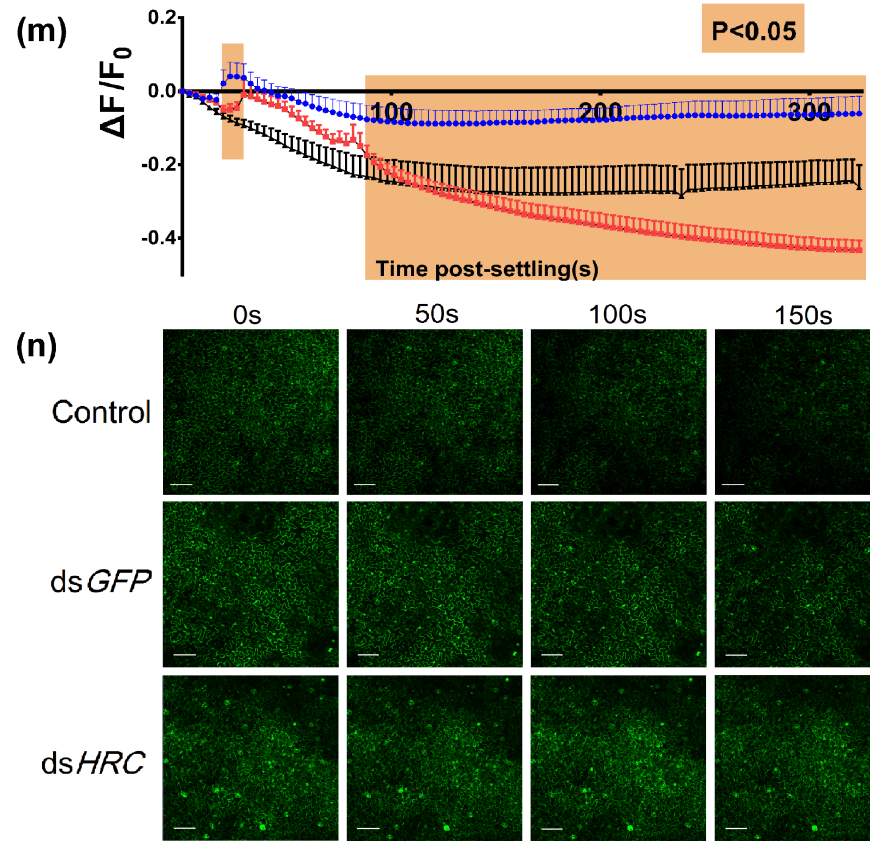

50 s

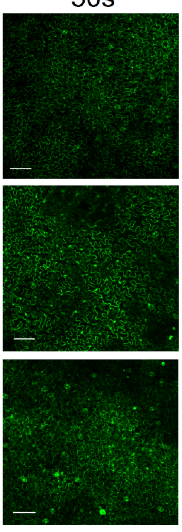

$100 \mathrm{~s}$

150s
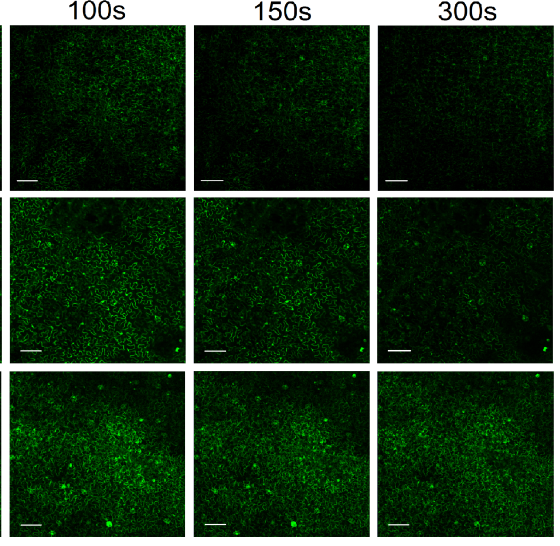
(a)

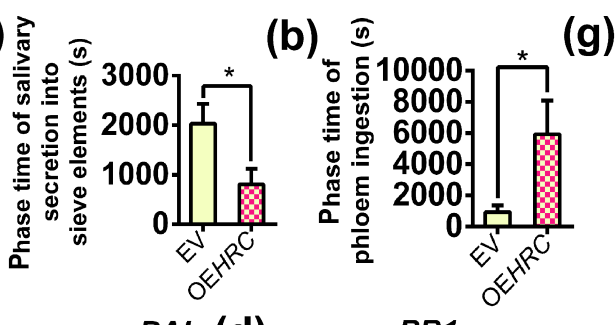

(c) 흐 $\quad P A L(d)$ 응

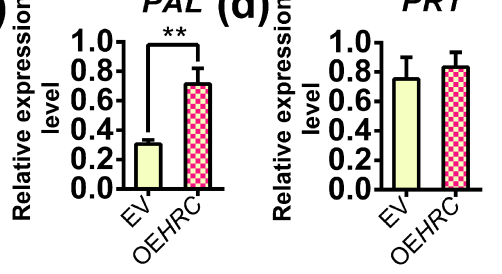

(e)

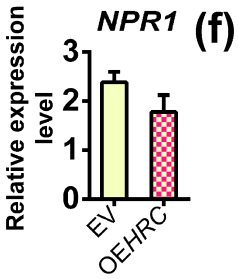

(i)

Control

EV

OEHRC
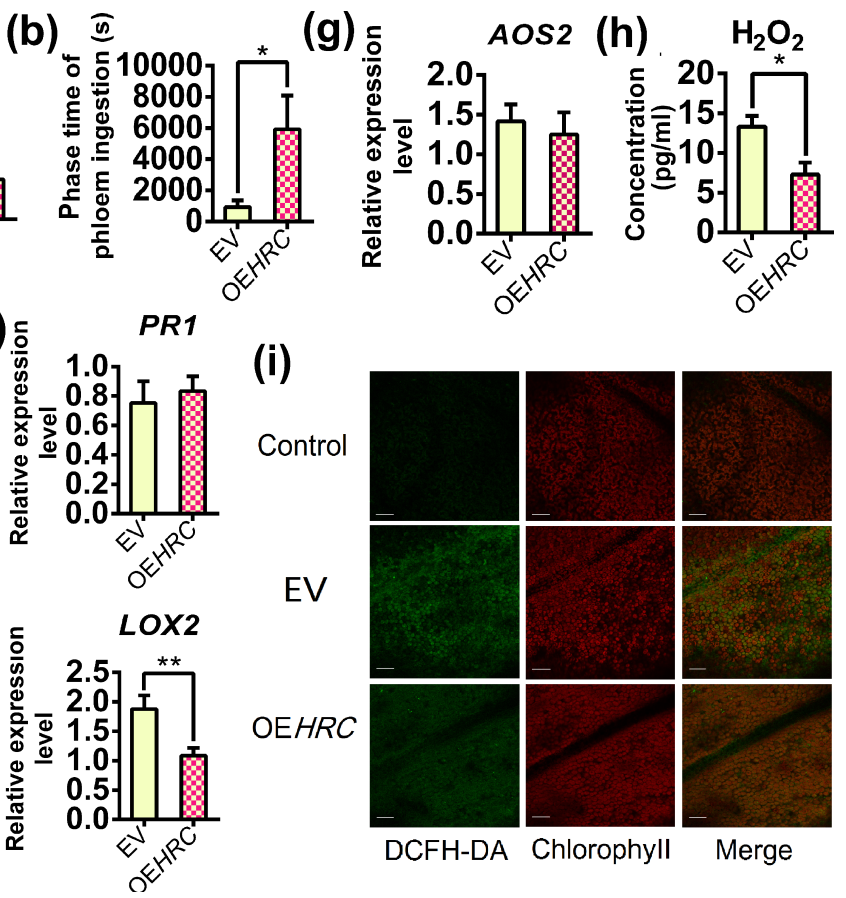

DCFH-DA Chlorophyll

Merge 
(a)

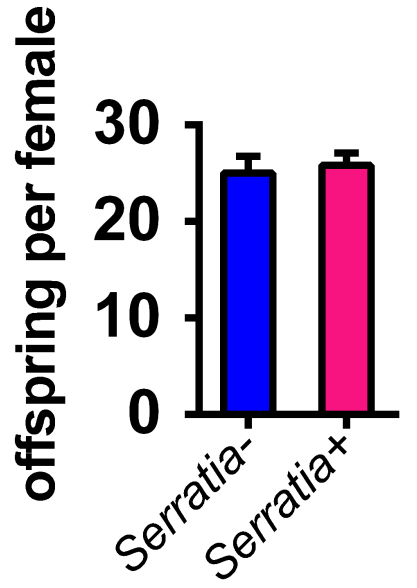

(b)

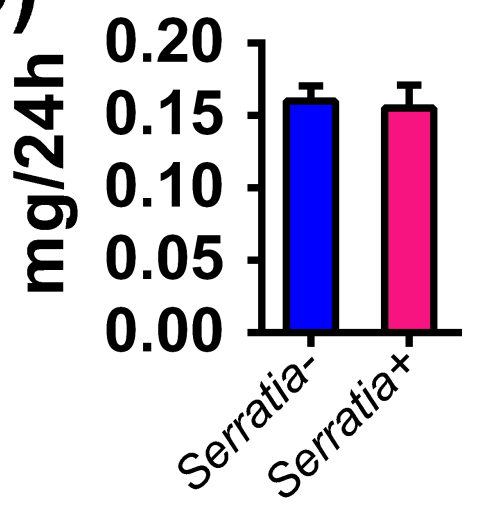

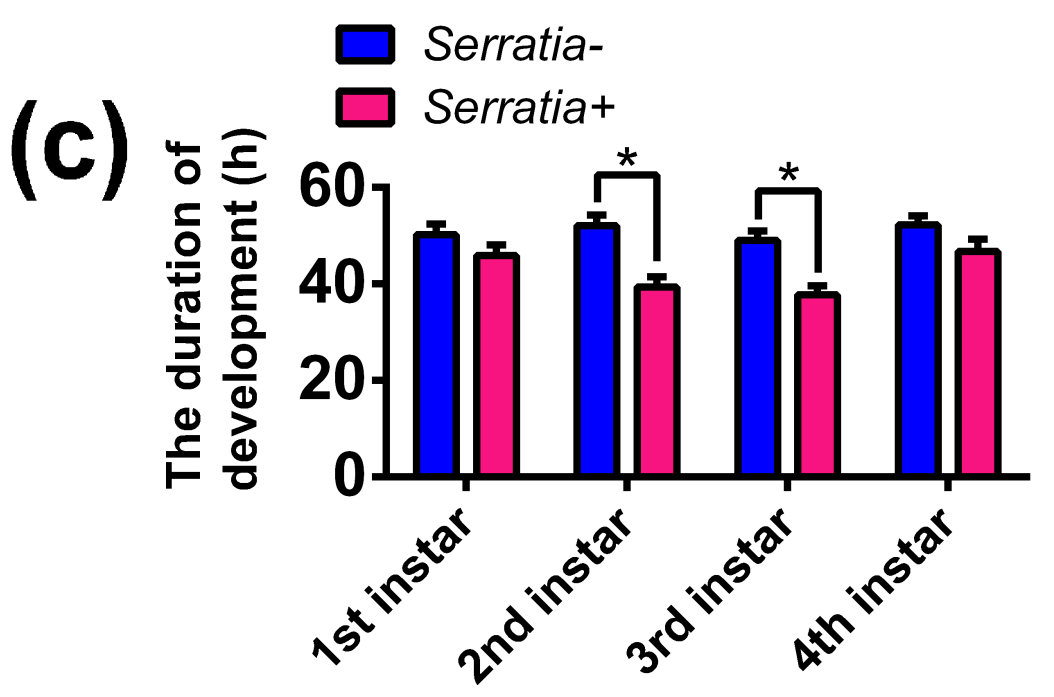

